\title{
$S$-dual inflation and the string swampland
}

\author{
Luis A. Anchordoqui, ${ }^{1,2,3}$ Ignatios Antoniadis, ${ }^{4,5}$ Dieter Lüst, ${ }^{6,7}$ and Jorge F. Soriano $\circledast^{1,2}$ \\ ${ }^{1}$ Department of Physics and Astronomy, Lehman College, City University of New York, \\ New York 10468, USA \\ ${ }^{2}$ Department of Physics, Graduate Center, City University of New York, New York 10016, USA \\ ${ }^{3}$ Department of Astrophysics, American Museum of Natural History, New York 10024, USA \\ ${ }^{4}$ Laboratoire de Physique Théorique et Hautes Énergies-LPTHE Sorbonne Université, \\ CNRS, 4 Place Jussieu, 75005 Paris, France \\ ${ }^{5}$ Institute for Theoretical Physics, KU Leuven, Celestijnenlaan 200D, B-3001 Leuven, Belgium \\ ${ }^{6}$ Max-Planck-Institut für Physik, Werner-Heisenberg-Institut, 80805 München, Germany \\ ${ }^{7}$ Arnold Sommerfeld Center for Theoretical Physics, Ludwig-Maximilians-Universität München, \\ 80333 München, Germany
}

(Received 25 March 2021; accepted 3 June 2021; published 22 June 2021)

\begin{abstract}
The swampland de Sitter conjecture in combination with upper limits on the tensor-to-scalar ratio $r$ derived from observations of the cosmic microwave background endangers the paradigm of slow-roll single-field inflation. This conjecture constrains the first and the second derivatives of the inflationary potential in terms of two $\mathcal{O}(1)$ constants $c$ and $c^{\prime}$. In view of these restrictions, we reexamine single-field inflationary potentials with $S$-duality symmetry, which ameliorate the unlikeliness problem of the initial condition. We compute $r$ at next-to-leading order in slow-roll parameters for the most general form of $S$-dual potentials and confront model predictions to constraints imposed by the de Sitter conjecture. We find that $c \sim \mathcal{O}\left(10^{-1}\right)$ and $c^{\prime} \sim \mathcal{O}\left(10^{-2}\right)$ can accommodate the 95\% C.L. upper limit on $r$. By imposing at least $50 e$-folds of inflation with the effective field theory description valid only over a field displacement $\mathcal{O}(1)$ when measured as a distance in the target space geometry, we further restrict $c \sim \mathcal{O}\left(10^{-2}\right)$, while the constraint on $c^{\prime}$ remains unchanged. We comment on how to accommodate the required small values of $c$ and $c^{\prime}$.
\end{abstract}

DOI: 10.1103/PhysRevD.103.123537

\section{INTRODUCTION}

Inflation is the leading paradigm for explaining the behavior of the quasi-de Sitter expansion in the very early Universe. Single-field inflationary models provide promising explanations to the cosmological horizon problem, the lack of topological defects, and the observed large-scale isotropy [1-3]. In addition, inflation provides a mechanism for generating small fluctuations in energy density, which could have seeded galactic structure formation [4-8] and are observed in the temperature anisotropies of the cosmic microwave background (CMB) $[9,10]$.

One of the main goals of modern $\mathrm{CMB}$ missions is to measure the tensor-to-scalar ratio $r$ accurately to constrain inflationary models. The combination of BICEP2/ Keck Array data with observations by Planck (TT,TE, EE+lowE + lensing) and baryon acoustic oscillation

Published by the American Physical Society under the terms of the Creative Commons Attribution 4.0 International license. Further distribution of this work must maintain attribution to the author(s) and the published article's title, journal citation, and DOI. Funded by SCOAP .
(BAO) significantly shrink the space of allowed inflationary cosmologies: $r<0.068$ at $95 \%$ C.L. [11,12]. Moreover, CMB data favor standard slow-roll single-field inflationary models with plateaulike potentials $V$, for which $V_{\phi \phi}<0$, over power-law potentials; here, $\phi$ is the dilaton or inflaton and $V_{\phi} \equiv d V / d \phi$ [13]. In this paper, we investigate slow-roll inflationary models within the context of the swampland program [14] and confront model predictions with experiment. We particularize the investigation to inflationary potentials satisfying $V_{\phi \phi}<0$ while being invariant under the $S$-duality constraint, $\phi \rightarrow-\phi$ [15], which is reminiscent of string theory [16,17].

The swampland program has been established to lay out a connection between quantum gravity and very-large-scaleultralow-energy astronomical observations. The string swampland comprises the set of (apparently) consistent effective field theories (EFTs) that cannot be completed into quantum gravity in the ultraviolet $[18,19]$. This rather abstract concept implies that if gravity were to be added into an EFT which is self-consistent up to a scale $E_{\text {self }}$, then the combined theory would exhibit a new limiting energy scale $E_{\text {swamp }}$, above which the theory must be modified if it is to become compatible with quantum gravity 
in the ultraviolet. When the energy relation $E_{\text {swamp }}<E_{\text {self }}<$ $E_{\mathrm{PI}}$ holds and $E_{\mathrm{swamp}}$ is below any characteristic energy scale involved in the theory, we say that the entire EFT belongs to the string swampland; $E_{\mathrm{Pl}}$ denotes the Planck energy scale. Guidance for a model building approach is provided by an ensemble of swampland conjectures [20-44]. There are two consequential conjectures which gathered immediate interest in the context of inflationary cosmology.

(i) Distance swampland conjecture.-This conjecture limits the field space of validity of any EFT by limiting the field excursion $\Delta \phi$ to be small when expressed in Planck units, namely, $\Delta \phi / M_{\mathrm{Pl}} \equiv \delta \lesssim \alpha \sim \mathcal{O}(1)$, where $M_{\mathrm{Pl}}=(8 \pi G)^{-1 / 2}$ is the reduced Planck mass [21-25].

(ii) de Sitter conjecture.-The gradient of the scalar potential $V$ must satisfy the lower bound,

$$
M_{\mathrm{Pl}} \frac{\left|V_{\phi}\right|}{V} \equiv \mathcal{C} \geq c,
$$

or else its Hessian must satisfy

$$
M_{\mathrm{Pl}}^{2} \frac{V_{\phi \phi}}{V} \equiv \mathcal{C}^{\prime} \leq-c^{\prime},
$$

where $c$ and $c^{\prime}$ are positive order-one numbers in Planck units $[23,28]$.

It has been noted that, while the distance conjecture by itself does not pose a significant challenge for single-field inflationary models (and corresponds observationally to a suppressed $r$ through the well-known Lyth bound [45]), the de Sitter conjecture is in direct tension with slow-roll inflationary potentials favored by CMB data [46-54]. The objective of our investigation is to analyze the status of single-field inflationary potentials with $S$-duality symmetry in the context of the swampland conjectures.

The remainder of the paper is structured as follows. In Sec. II, we first provide an overview of the equations of motion in single-field slow-roll inflation and introduce the definition of the slow-roll parameters. After that, to make the connection with experiment, we compute the scalar spectral index $n_{s}$ and the tensor-to-scalar ratio $r$ at next-toleading order (NLO) in slow-roll parameters. In Sec. III, we examine the subtleties of model building while imposing constraints which depend on multiple slow-roll parameters focusing attention on $S$-dual symmetric inflationary potentials. We summarize the generalities of these potentials and confront model predictions to the CMB observables $n_{s}$ and $r$. In Sec. IV, we investigate the ambiguity on the definition of the slow-roll parameters and explore whether this uncertainty can help ameliorate the tension between single-field inflationary models and the de Sitter swampland conjecture. The paper wraps up with some conclusions presented in Sec. V.

\section{CONSTRAINTS ON $r$ AT NLO IN SLOW-ROLL PARAMETERS}

The essential property of nearly all crowned inflationary models is a period of slow-roll evolution of $\phi$ during which its kinetic energy remains always much smaller than its potential energy. The equation of motion for the canonical homogeneous inflaton field is

$$
\ddot{\phi}+3 H \dot{\phi}+V_{\phi}=0,
$$

where $H=\dot{a} / a$ is the Hubble parameter and the dot denotes derivative with respect to the cosmic time. The slow-roll conditions

$$
\frac{1}{2} \dot{\phi}^{2} \ll|V|
$$

and

$$
\left|\frac{\ddot{\phi}}{3 H \dot{\phi}}\right| \ll 1
$$

imply

$$
\epsilon \equiv \frac{M_{\mathrm{Pl}}^{2}}{2}\left(\frac{V_{\phi}}{V}\right)^{2} \ll 1
$$

and

$$
\eta \equiv M_{\mathrm{Pl}}^{2}\left[\frac{V_{\phi \phi}}{V}-\frac{1}{2}\left(\frac{V_{\phi}}{V}\right)^{2}\right] \ll 1,
$$

respectively $[55,56] .{ }^{1}$ The Friedmann relation incorporating slow roll is given by

$$
H(\phi) \simeq \sqrt{\frac{V}{3 M_{\mathrm{Pl}}^{2}}} .
$$

At the end of slow roll, $\phi$ falls into the core of the potential and oscillates rapidly around the minimum, ultimately leading to the reheating period. The amount of inflationary expansion within a given timescale is generally parametrized in terms of the number of $e$-foldings that occur as the scalar field rolls from a particular value $\phi$ to its value $\phi_{e}$ when inflation ends:

$$
N\left(\phi \rightarrow \phi_{e}\right)=-\frac{1}{M_{\mathrm{Pl}}^{2}} \int_{\phi}^{\phi_{e}} \frac{V}{V_{\phi}} d \phi,
$$

with $\epsilon\left(\phi_{e}\right)=1$ [58]. The de Sitter conjecture bounds the integrand above. Around a minimum of the potential without changes in the curvature

\footnotetext{
${ }^{1}$ The definitions of the slow-roll parameters vary; we follow the conventions of Ref. [57].
} 


$$
\begin{aligned}
N\left(\phi \rightarrow \phi_{e}\right) & =\frac{1}{M_{\mathrm{Pl}}^{2}} \int_{\min \left(\phi, \phi_{e}\right)}^{\max \left(\phi, \phi_{e}\right)}\left|\frac{V}{V_{\phi}}\right| d \phi \leq \frac{\left|\phi_{e}-\phi\right|}{M_{\mathrm{Pl}}^{2}} \max _{\left[\phi, \phi_{e}\right]}\left|\frac{V}{V_{\phi}}\right| \\
& =\frac{\left|\phi_{e}-\phi\right|}{M_{\mathrm{Pl}}^{2}}\left|\frac{V}{V_{\phi}}\right|_{\phi}
\end{aligned}
$$

as $V / V_{\phi} \sim 1 / \sqrt{\epsilon}$ grows as the field moves away from the minimum. Using the de Sitter conjecture bound, this can be written more compactly as [51]

$$
\frac{\Delta \phi}{M_{\mathrm{Pl}}}>c N \text {. }
$$

To make contact with experiment, we calculate $r$ at NLO in slow-roll parameters. We begin by parametrizing the scalar

$$
\mathcal{P}_{s}=A_{s}\left(\frac{k}{k_{*}}\right)^{n_{s}-1+\frac{1}{2} \alpha_{s} \ln \left(\frac{k}{k_{*}}\right)+\cdots}
$$

and tensor

$$
\mathcal{P}_{t}=A_{t}\left(\frac{k}{k_{*}}\right)^{n_{t}+\frac{1}{2} \alpha_{t} \ln \left(\frac{k}{k_{*}}\right)+\cdots}
$$

power spectra, where the spectral indices and their running (included here for completeness only) are given by

$$
\begin{aligned}
n_{s} \simeq 1 & -4 \epsilon+2 \eta+\left(\frac{10}{3}+4 \mathcal{B}\right) \epsilon \eta-(6+4 \mathcal{B}) \epsilon^{2} \\
& +\frac{2}{3} \eta^{2}-\frac{2}{3}(3 \mathcal{B}-1)\left(2 \epsilon^{2}-6 \epsilon \eta+\xi^{2}\right), \\
n_{t} \simeq & -2 \epsilon+\left(\frac{8}{3}+4 \mathcal{B}\right) \epsilon \eta-\frac{2}{3}(7+6 \mathcal{B}) \epsilon^{2}, \\
\alpha_{s} \equiv & \frac{d n_{s}}{d \ln k} \simeq-8 \epsilon^{2}+16 \epsilon \eta-2 \xi^{2}, \\
\alpha_{t} \equiv & \frac{d n_{t}}{d \ln k} \simeq-4 \epsilon(\epsilon-\eta),
\end{aligned}
$$

and where $\mathcal{B}=\gamma_{\mathrm{E}}+\ln 2-2 \approx-0.7296$ and

$$
\xi^{2} \equiv \frac{M_{\mathrm{Pl}}^{4} V_{\phi} V_{\phi \phi \phi}}{V^{2}}
$$

is the third slow-roll parameter [59]. The NLO amplitudes are related to $\epsilon, \eta$, and $V$ by

$$
\begin{aligned}
& \mathcal{A}_{s} \simeq \frac{V}{24 \pi^{2} M_{\mathrm{Pl}}^{4} \epsilon}\left[1-(4 \mathcal{B}+1) \epsilon+\left(2 \mathcal{B}-\frac{2}{3}\right) \eta\right], \\
& \mathcal{A}_{t} \simeq \frac{V}{6 \pi^{2} M_{\mathrm{Pl}}^{4}}\left[1-\left(2 \mathcal{B}+\frac{5}{3}\right) \epsilon\right] .
\end{aligned}
$$

All in all, the ratio of the NLO amplitudes of the spectra is given by

$$
r \equiv \frac{\mathcal{A}_{t}}{\mathcal{A}_{s}} \simeq 16 \epsilon+32\left(\mathcal{B}-\frac{1}{3}\right) \epsilon(\epsilon-\eta)
$$

Substituting Eq. (1) into Eq. (18), we reproduce the wellknown constraint at LO in slow-roll parameters:

$$
r \simeq 16 \epsilon \equiv 8 \mathcal{C}^{2} \Rightarrow \mathcal{C} \simeq \sqrt{r / 8} \lesssim 0.09,
$$

where we have taken $r$ to saturate the $95 \%$ C.L. upper limit. A comparison of this upper limit with the lower limit in Eq. (1) has called into question whether slow-roll single-field inflationary models could live on the swampland [46-54]. Using the upper value of the measured $1 \sigma$ range, $n_{s}=0.9658 \pm 0.0040$ [11], a combined limit on $\mathcal{C}$ and $\mathcal{C}^{\prime}$ can be derived substituting Eqs. (1) and (2) into the expression of the scalar spectral index (14). At LO,

$$
n_{s} \simeq 1-2 \mathcal{C}^{2}+2 \eta .
$$

The allowed region of the $n_{s}-r$ plane at LO has been reported in Ref. [50]. We can visualize the modification of the NLO bounds on $\mathcal{C}$ and $\mathcal{C}^{\prime}$ posed by the data in Fig. 1 in a model-independent way, to the degree that the $\xi^{2}$ term in the expansion for $n_{s}$ in Eq. (14) is negligible. This alone suggests a certain degree of incompatibility between observations and the de Sitter conjecture. The inclusion of a nonzero $\xi$ would slightly reduce the tension with $c^{\prime}$, displacing down the contours of Fig. 1 (right) but leaving them almost unchanged along the $\mathcal{C}$ direction.

In the next section, we will particularize our study to inflationary potentials with $S$-duality symmetry. In particular, we will explore the relevance of the distance swampland conjecture, which cannot be explored in a model-independent way at any order.

\section{III. $S$ DUALITY STRIKES AGAIN}

Dualities within gauge theories are striking, as they relate a strongly coupled field theory to a weakly coupled one, and thereby they are handy for evaluating a theory at strong coupling, where perturbation theory breaks down, by translating it into its dual description with a weak coupling constant; ergo, dualities point to a single quantum system which has two classical limits. The $U(1)$ gauge theory on $\mathbb{R}^{4}$ is known to possess an electric-magnetic duality symmetry that inverts the coupling constant and extends to an action of $S L(2, \mathbb{Z})$ [15]. There are also many examples of $S$ duality in string theory $[16,17]$. In this section, we examine potentials which are invariant under the $S$-duality constraint and confront them with experiment. Herein, we do not attempt a full association with a particular string vacuum but simply regard the self-dual constraint as a relic of string physics in inflationary cosmology. We adopt a phenomenological approach to expand the inflationary potential in terms of a generic form 

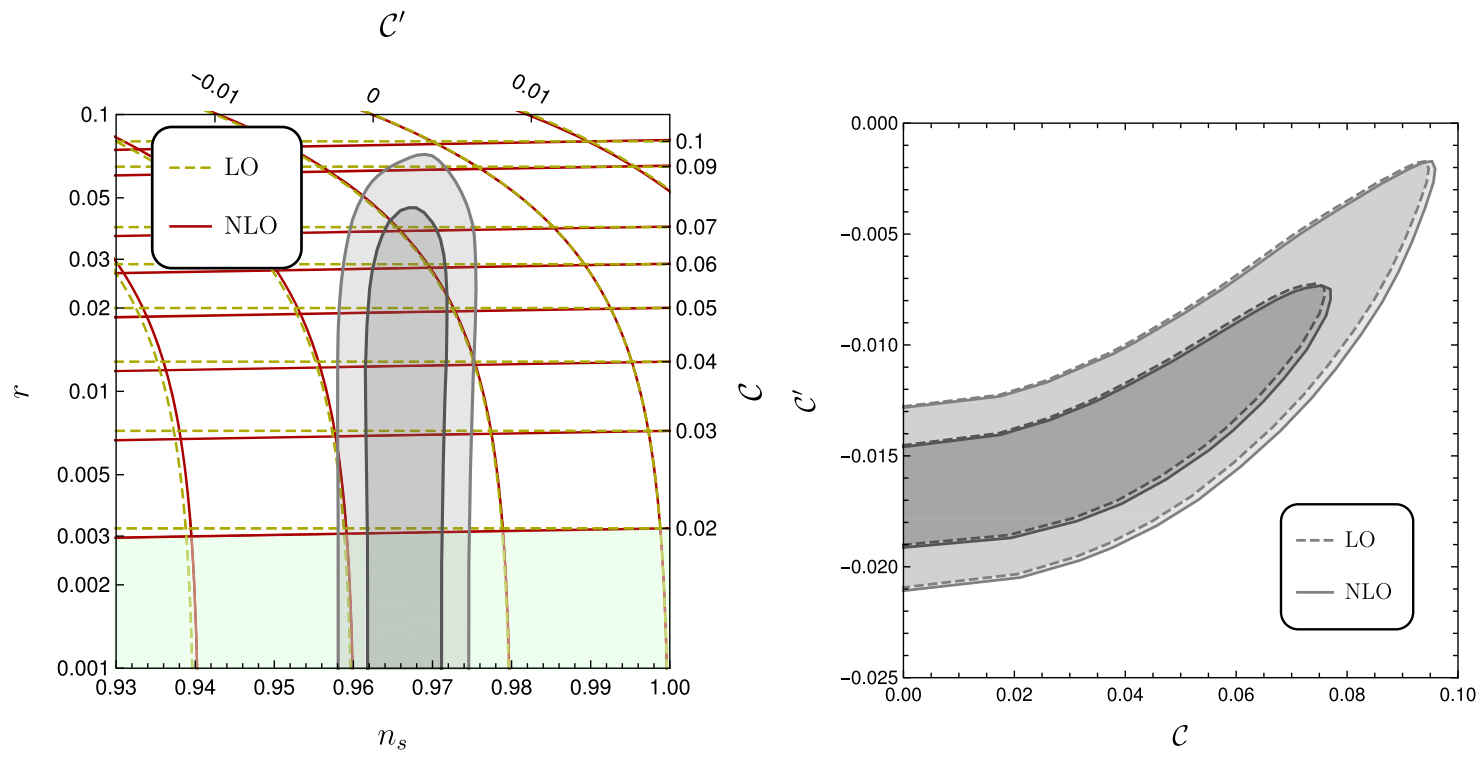

FIG. 1. Relation between $\left(r, n_{s}\right)$ and $\left(\mathcal{C}, \mathcal{C}^{\prime}\right)$ at LO and NLO, together with the bound (11) (shaded region) for $N=50$ and $\Delta \phi=M_{\mathrm{Pl}}$ (left), and experimental constraints on $\left(\mathcal{C}, \mathcal{C}^{\prime}\right)$ at LO and NLO from TT, TE, EE + lowE + lensing + BK15 + BAO data $[11]($ right).

satisfying the $S$-duality constraint, and then the determination of the expansion coefficients is data driven.

For a real scalar field $\phi$, the $S$-duality symmetry is $\phi \rightarrow-\phi$ (or, alternatively, $g \rightarrow 1 / g$, with $g \sim e^{\phi / M_{\mathrm{Pl}}}$ ). In case there is an imaginary part, i.e., an axion, then the $S$ duality group is extended to the modular group $S L(2, \mathbb{Z})$. The $S$-duality constraint forces a particular functional form on the inflationary potential: $V(\phi)=f\left[\cosh \left(\kappa \phi / M_{\mathrm{Pl}}\right)\right]$, where $\kappa$ is a constant [60].

A compelling property of inflationary potentials featuring $S$-duality symmetry is that they resolve the "unlikeliness problem," which is typical of plateaulike potentials, e.g.,

$$
V_{1}(\phi)=\frac{V_{0}^{(1)}}{M_{\mathrm{Pl}}^{4}}\left(\phi^{2}-\phi_{0}^{2}\right)^{2},
$$

where $V_{0}$ and $\phi_{0}$ are free parameters [61]. Note that the plateau region satisfies $\phi \ll \phi_{0}$ terminating at the local minimum, and for large values of $\phi$ the potential grows as a power law $\sim V_{0}\left(\phi / M_{\mathrm{Pl}}\right)^{4}$. This means that we have two paths to reach the minimum of the potential: by slow roll along the plateau or by slow roll from the power-law side of the minimum. The problem appears because the path from the power-law side requires less fine-tuning of parameters, has inflation occurring over a much wider range of $\phi$, and produces exponentially more inflation, but still CMB data prefer the unlikely path along the plateau.

The simplest $S$ self-dual form,

$$
V_{2}(\phi)=V_{0}^{(2)} \operatorname{sech}\left(\frac{\kappa \phi}{M_{\mathrm{Pl}}}\right)
$$

solves the unlikeliness problem because it has no powerlaw wall. Moreover, it is easily seen that for Eqs. (21) and (22) the slow-roll parameters $\epsilon$ and $\eta$ are of the scale $\left(\phi_{0} / M_{\mathrm{Pl}}\right)^{2} \sim \kappa^{-1}$ and, thus, have similar inflationary growths; see Fig. 2. However, for Eq. (21), the slow-roll parameters $\epsilon$ and $\eta$ grow fast near the end of inflation $\left(\phi \sim \phi_{0}\right)$, but, for the $S$ self-dual form, $\epsilon$ and $\eta$ remain small, because the potential has no local minimum. Thereby, $\phi$ cannot exit the inflationary period.

To describe $S$-dual potentials for which inflation ends, we adopt a polynomial expression in the sech function. Without loss of generality, we can write it as

$$
V(\phi)=V_{0} \sum_{n=0}^{N} a_{n} \operatorname{sech}^{n}\left(\frac{\kappa \phi}{M_{\mathrm{Pl}}}\right),
$$

under the condition that $\sum_{i} a_{i}=1$, to ensure that $V_{0}=V(0)$. Here, the normalization constant $V_{0}$ and the expansion coefficients $a_{n}$ are determined empirically by matching experimental constraints. To determine the coefficients $a_{n}$, we demand

(i) $N\left(\phi_{*} \rightarrow \phi_{e}\right) \simeq 60$, with $\phi_{*}$ the field value when the $k_{*}$ scale crosses the horizon, $k_{*}=a H$;

(ii) the NLO expression of $r$ given in Eq. (18) to satisfy the $95 \%$ C.L. upper limit, i.e., $r<0.069$ [11];

(iii) the NLO expression of the scalar spectral index $n_{s}$ given in Eq. (14) to match the upper end of the measured $1 \sigma$ value, $n_{s} \simeq 0.9698$ [11].

The phenomenological expression in Eq. (22) could develop a minimum to support dissipative oscillations at the cessation of the slow roll and reheating and resolves the unlikeliness problem. In order to analyze 

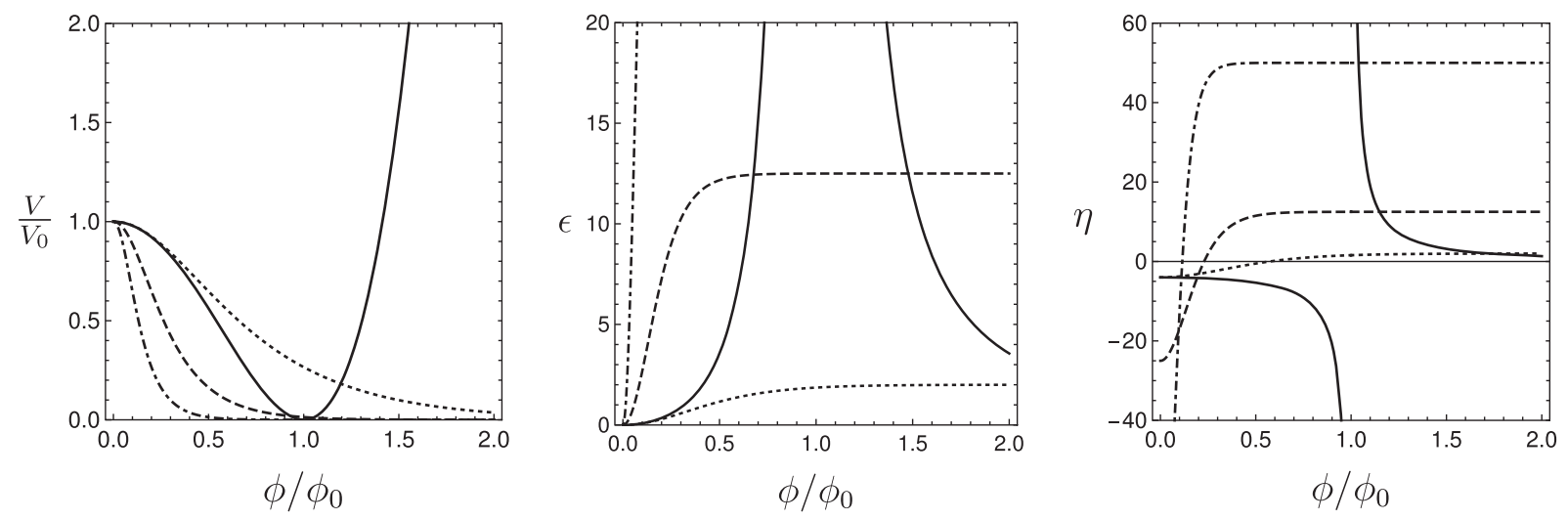

FIG. 2. Potential and slow-roll functions for the potentials defined by Eqs. (21) (solid line) and (22) with $\kappa=2$ (dotted line), $\kappa=5$ (dashed line), and $\kappa=10$ (dot-dashed line).

the model, it is convenient to define $y=\operatorname{sech}\left(\kappa \phi / M_{\mathrm{Pl}}\right)$ and $V(\phi)=V_{0} f(y)$, with

$$
f(y)=\sum_{n=0}^{N} a_{n} y^{n} .
$$

Without conflicting with $S$ duality, we restrict ourselves here to $\phi>0$ to guarantee a bijection between $y$ and $\phi$. Note that $y \in[0,1]$ as $\phi \in[0, \infty)$. It is then easy to see that

$$
\begin{aligned}
V_{\phi} & =-V_{0} \frac{\kappa}{M_{\mathrm{Pl}}} y \sqrt{1-y^{2}} f^{\prime}(y), \\
V_{\phi \phi} & =V_{0}\left(\frac{\kappa}{M_{\mathrm{Pl}}}\right)^{2}\left[y^{2}\left(1-y^{2}\right) f^{\prime \prime}(y)+y\left(1-2 y^{2}\right) f^{\prime}(y)\right],
\end{aligned}
$$

and

$$
\begin{aligned}
V_{\phi \phi \phi}= & V_{0}\left(\frac{\kappa}{M_{\mathrm{Pl}}}\right)^{3} y \sqrt{1-y^{2}}\left[-y^{2}\left(1-y^{2}\right) f^{\prime \prime \prime}(y)\right. \\
& \left.-3 y\left(1-2 y^{2}\right) f^{\prime \prime}(y)-\left(1-6 y^{2}\right) f^{\prime}(y)\right],
\end{aligned}
$$

which allow one to obtain analytical expressions for $\epsilon, \eta$, and $\xi$.

Nontrivial potentials occur for $N \geq 2$. Here, we study the polynomial form in Eq. (22) at lowest order, i.e., $f(y)=a_{0}+a_{1} y+a_{2} y^{2}$. From the initially four model parameters $\left(V_{0}, a_{1}, a_{2}, a_{3}\right)$, the normalization condition $V(0)=V_{0}$ [or $f(1)=1$ ] allows one to remove one of them. The potential has a minimum, at which we can impose $V=0$, removing another constant. It is easily seen that in this case $f$ can be rewritten as

$$
f(y)=\left(\frac{y-\beta}{1-\beta}\right)^{2},
$$

where $\beta \in(0,1)$ is the position of the minimum. This corresponds to a potential

$$
V(\phi)=V_{0}\left[\frac{\operatorname{sech}\left(\kappa \frac{\phi}{M_{\mathrm{Pl}}}\right)-\operatorname{sech}\left(\kappa \frac{\phi_{0}}{M_{\mathrm{Pl}}}\right)}{1-\operatorname{sech}\left(\kappa \frac{\phi_{0}}{M_{\mathrm{Pl}}}\right)}\right]^{2},
$$

where $\beta=\operatorname{sech}\left(\kappa \phi_{0} / M_{\mathrm{Pl}}\right)$. A point worth noting at this juncture is that the expansion of Eq. (23) is not hierarchical; i.e., the coefficients $a_{n}$ should not necessarily become smaller and smaller with larger $n$. Our choice is based on the complexity of the model, in which larger $N$ potentials would contain more free parameters and, under some conditions, more maxima or minima. Note that an identification of Eq. (24) with Eq. (26) allows one to see that $a_{1} / a_{0}=-2 / \beta$ and $a_{2} / a_{1}=-1 / 2 \beta$ and the hierarchy, if existing, is contingent on the position of the minimum of the field $\phi_{0}$ and on $\kappa$.

In Fig. 3, we show a comparison between the model described by Eq. (27) and the one introduced in Eq. (21). It is important to note that, for small $\kappa$, both potentials become similar. Indeed, up to $\mathcal{O}\left(\kappa^{3}\right)$ terms,

$$
V(\phi) \approx \frac{V_{0}}{V_{0}^{(1)}}\left[\left(\frac{M_{\mathrm{Pl}}}{\phi_{0}}\right)^{4}-\frac{5}{6} \frac{\kappa^{2} M_{\mathrm{Pl}}^{2}}{\phi_{0}^{2}} \frac{\phi^{2}}{\phi_{0}^{2}}\right] V_{1}(\phi) .
$$

The zeroth-order difference may be absorbed in the normalization of the potentials, so the potentials can be made almost identical ${ }^{2}$ for

$$
\phi \lesssim \sqrt{\frac{6}{5}} \frac{M_{\mathrm{Pl}}}{\kappa} .
$$

Then, only a relatively large $\kappa$ would produce substantial differences between both models if we want to avoid using

\footnotetext{
${ }^{2}$ To the extent that their overall normalizations are irrelevant, as is the case for all quantities derived from the slow-roll parameters or the number of inflation $e$-folds.
} 

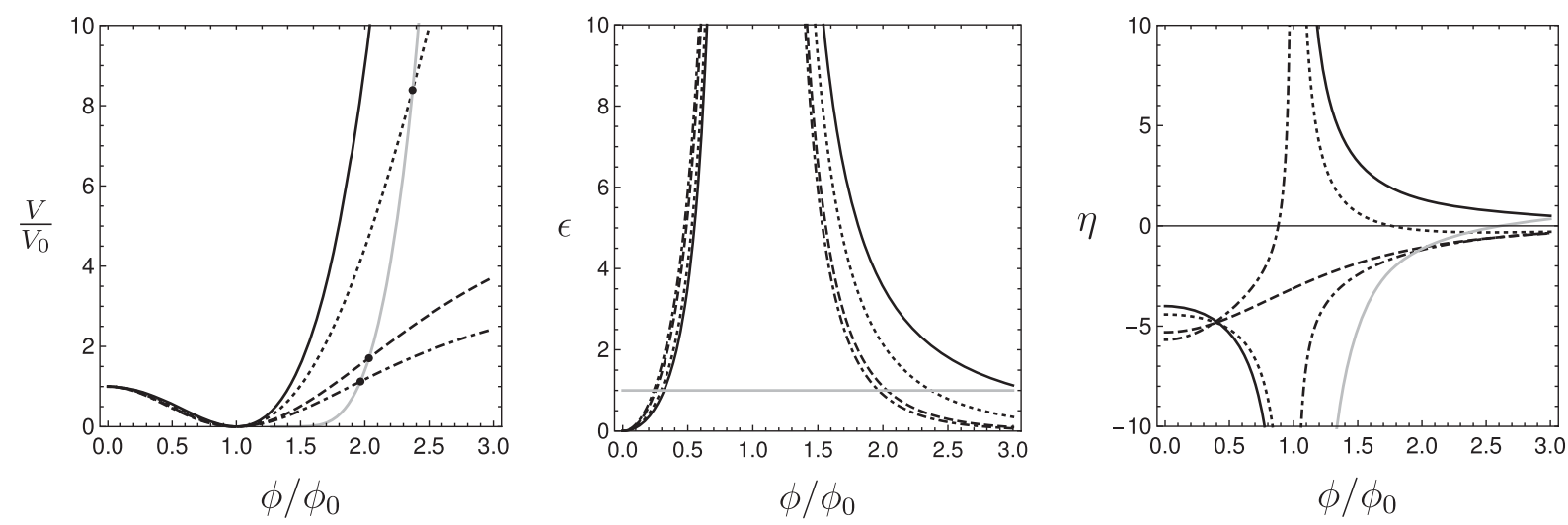

FIG. 3. Potential and slow-roll functions for the potentials defined by Eqs. (21) (solid line) and (27) with $\kappa=1 / 2$ (dotted line), $\kappa=\ln (1+\sqrt{2})$ (dashed line), and $\kappa=1$ (dot-dashed line). The gray lines are the end of inflation $(\epsilon=1)$.

highly trans-Planckian fields. For larger fields, the differences are more obvious, as $V_{1}$ grows indefinitely while

$$
\lim _{\phi \rightarrow \infty} V(\phi)=\frac{V_{0}}{4} \operatorname{csch}^{4}\left(\frac{\kappa \phi_{0}}{2 M_{\mathrm{Pl}}}\right)
$$

The slow-roll parameters can be now obtained from Eqs. (25) and (26) and are given by

$$
\begin{aligned}
& \epsilon=\frac{2 \kappa^{2} y^{2}\left(1-y^{2}\right)}{(y-\beta)^{2}}, \\
& \eta=\frac{2 \kappa^{2} y\left(1-2 y^{2}\right)}{y-\beta},
\end{aligned}
$$

and

$$
\xi=\frac{4 \kappa^{4} y^{2}\left(y^{2}-1\right)\left(\beta+2 y\left(6 y^{2}-3 \beta y-2\right)\right)}{(y-\beta)^{3}}
$$

these can be easily combined with Eqs. (14) and (18) to explore the parameter space in terms of $n_{s}, r$, and $N$. The first step in that direction requires to find out the condition (s) for slow roll to end. The potential under consideration allows for two types of slow-roll inflation: (i) one in which $\phi$ rolls down the potential toward a minimum at larger values and (ii) one in which a large field rolls down the potential toward smaller values. The condition $\epsilon=1$ may be rewritten as the quartic polynomial equation

$$
y^{4}+\frac{1-2 \kappa^{2}}{2 \kappa^{2}} y^{2}-\frac{\beta}{\kappa^{2}} y+\frac{\beta^{2}}{2 \kappa^{2}}=0,
$$

which has, in principle, four complex roots, which may be obtained following Ferrari's method [62]. For the polynomial $y^{4}+q y^{2}+r y+s$, the roots are found to be

$$
y=\sigma_{1} \sqrt{\frac{u}{2}}+\sigma_{2} \sqrt{-\frac{u}{2}-\frac{q}{2}-\sigma_{1} \frac{r}{2 \sqrt{2 u}}},
$$

where $\sigma_{1}$ and $\sigma_{2}$ are two independent signs that generate the four solutions and $u$ is a solution to the cubic equation $u^{3}+q u^{2}+\left(q^{2} / 4-s\right) u-r^{2} / 8=0$. This can be reduced by a change of variables $u=v-q / 3$ to a depressed cubic equation $v^{3}-\left(s+q^{2} / 12\right) v-\left(2 q^{3}+27 r^{2}-72 q s\right) / 216=0$. Such an equation, generally $v^{3}+a v+b=0$, has a solution given by Cardano's formula $v=\sqrt{3} \mathcal{A}_{+}+\sqrt{3} \mathcal{A}_{-}$, with $\mathcal{A}_{ \pm}=-b / 2 \pm \sqrt{\Delta}$ and $\Delta=(a / 3)^{3}+(b / 2)^{2}$, which, reverting the changes of variables, is

$\Delta=-\frac{\beta^{2}}{2^{8} 3^{3} \kappa^{8}}\left[32 \kappa^{2} \beta^{4}+\left(1-8 \kappa^{2}\left(5+4 \kappa^{2}\right) \beta^{2}-\left(1-2 \kappa^{2}\right)^{3}\right]\right.$.

It is clear that the nature of the solutions depends on the sign of $\Delta$, which is unconstrained. The lines $\Delta=0$, which separate both regions, may be solved explicitly for $\beta$. Out of the four possible solutions, only

$$
\beta_{0}(\kappa)=\frac{1}{8} \sqrt{-\frac{\left(16 \kappa^{2}+1\right)^{3 / 2}}{\kappa^{2}}+32 \kappa^{2}-\frac{1}{\kappa^{2}}+40}
$$

is in the $\beta \in[0,1]$ and $\kappa>0$ region. The previous equation determines a limit in the $\kappa>1 / \sqrt{2}$ region, the value below which $\beta_{0}$ becomes complex. Moreover, $\kappa=\frac{1}{2} \sqrt{11+5 \sqrt{5}}$ marks the point at which $\beta=1$. For $\beta>\beta_{0}(\kappa), \Delta>0$. Then $\kappa<1 / \sqrt{2} \Rightarrow \Delta>0$ and $\kappa>\frac{1}{2} \sqrt{11+5 \sqrt{5}} \Rightarrow$ $\Delta<0$. Conversely, $\Delta<0 \Rightarrow \kappa>1 / \sqrt{2}$ and $\Delta>0 \Rightarrow$ $\kappa<\frac{1}{2} \sqrt{11+5 \sqrt{5}}$. For $1 / \sqrt{2}<\kappa<\frac{1}{2} \sqrt{11+5 \sqrt{5}}$, the curve $\beta_{0}(\kappa)$ separates both regions.

$\Delta>0$. - In this case, $\mathcal{A}_{ \pm}$is real, and $u$ may be written directly as $u=\sqrt[3]{\mathcal{A}_{+}}+\sqrt[3]{\mathcal{A}_{-}}-q / 3$. It it possible to 
see that $\mathcal{A}_{ \pm}>0$ over the region where $\Delta>0$. Moreover, even if $q>0$ somewhere, $u>0$ everywhere. If $\sigma_{1}=+1, y$ is real and $\sigma_{2}$ generates both solutions. The case $\sigma_{1}=-1$ corresponds to complex solutions in all range where $\Delta>0$. Then, the two solutions of interest here are given by

$$
y_{ \pm}=\sqrt{\frac{u}{2}} \pm \sqrt{-\frac{u}{2}-\frac{q}{2}-\frac{r}{2 \sqrt{2 u}}}
$$

and

$$
\begin{aligned}
u= & \left(-\frac{b}{2}+\sqrt{\left(\frac{b}{2}\right)^{2}+\left(\frac{a}{3}\right)^{3}}\right)^{\frac{1}{3}} \\
& +\left(-\frac{b}{2}-\sqrt{\left(\frac{b}{2}\right)^{2}+\left(\frac{a}{3}\right)^{3}}\right)^{\frac{1}{3}}-\frac{q}{3} .
\end{aligned}
$$

$\Delta<0$.- In this case, the solution to the cubic equation contains complex terms, and it becomes convenient to define $\mathcal{A}_{ \pm}=\mathcal{A} \exp ( \pm i \theta)$, where $\mathcal{A} \equiv\left|\mathcal{A}_{ \pm}\right|=\sqrt{b^{2} / 4-\Delta}=$ $\sqrt{-(a / 3)^{3}}$ and $\theta=2 \arctan [\sqrt{-\Delta} /(\mathcal{A}-b / 2)]$, which allows one to write $v=2 \mathcal{A}^{\frac{1}{3}} \cos (\theta / 3)$ and see that it is explicitly real. Moreover, as $\theta$ ranges in $[0, \pi], \cos (\theta / 3)$ is not negative. This, together with the fact that $q<0$ if $\kappa>1 / \sqrt{2}$, makes $u$ positive as well. In this region, though, both values of $\sigma_{1}$ generate real solutions. It is clear that $\sigma_{1}=\sigma_{2}=-1$ would yield a negative solution, irrelevant in this case. Further investigation reveals that the other solution with $\sigma_{1}=-1$ yields negative solutions as well. The other solutions are always contained in $[0,1]$. Then, the solutions in this case are the same $y_{ \pm}$defined in Eq. (36), where now $u$ is better expressed involving real numbers only as

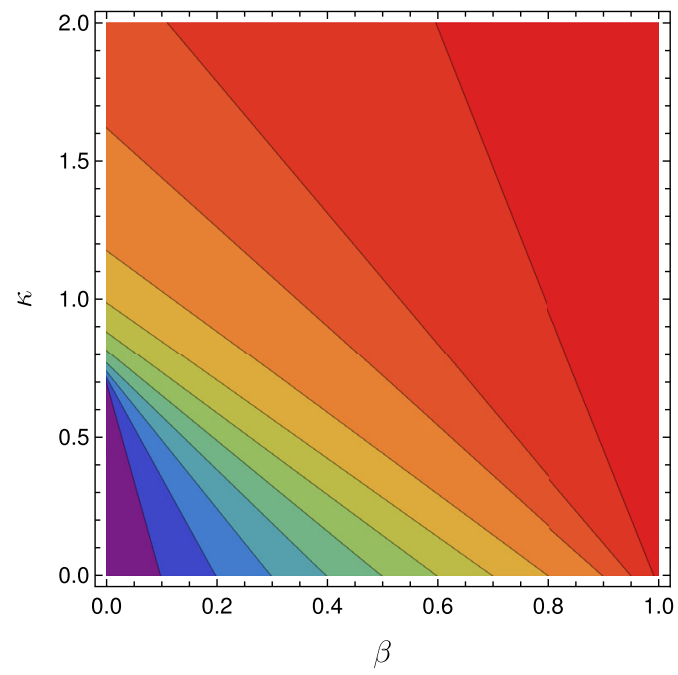

(a) Solution $y_{+}$from (36a). $u=2 \sqrt{-\frac{a}{3}} \cos \left[\frac{2}{3} \arctan \left(\frac{\sqrt{-(b / 2)^{2}-(a / 3)^{3}}}{\sqrt{-(a / 3)^{3}}-b / 2}\right)\right]-\frac{q}{3}$.

The parameters $a, b$, and $q$ used in all solutions above are, respectively,

$$
a=-\frac{\beta^{2}}{2 \kappa^{2}}-\frac{\left(1-2 \kappa^{2}\right)^{2}}{48 \kappa^{4}},
$$

$$
b=\frac{\beta^{2}\left(1-2 \kappa^{2}\right)}{12 \kappa^{4}}-\frac{\beta^{2}}{8 \kappa^{4}}-\frac{\left(1-2 \kappa^{2}\right)^{3}}{864 \kappa^{6}},
$$

and

$$
q=\frac{1-2 \kappa^{2}}{2 \kappa^{2}}
$$

The solutions $y_{ \pm}$to the end of inflation equation $\epsilon=1$ are shown in Fig. 4. We recall here that $y_{-}$corresponds to a solution for smaller $y$ (larger $\phi$ ) and $y_{+}$to larger $y$ (smaller $\phi)$. We define $y_{ \pm}=\operatorname{sech}\left(\kappa \phi_{ \pm} / M_{\mathrm{Pl}}\right)$.

We can now proceed with our analysis noting that, besides the values of $\beta$ and $\kappa$, which determine the end of inflation, there is still freedom in choosing the value of the field at the scale that corresponds to the experimental values. We call this $\phi_{* \pm}$ and define $\delta_{ \pm}= \pm\left(\phi_{ \pm}-\phi_{* \pm}\right) / M_{\mathrm{Pl}}$. We recall that the distance swampland conjecture demands that $\delta_{ \pm} \lesssim \mathcal{O}(1)$. In terms of our model, when we are considering large fields (minus signs above), the value of $\phi_{*-}$ is unbounded and $\delta_{-}$could take any positive value. Nevertheless, for small fields (plus signs above), $\delta_{+}$is constrained so that $\phi_{*+}>0$, which means $\delta_{+}<\phi_{+} / M_{\mathrm{Pl}}$. All in all, the corresponding values for $y$ are

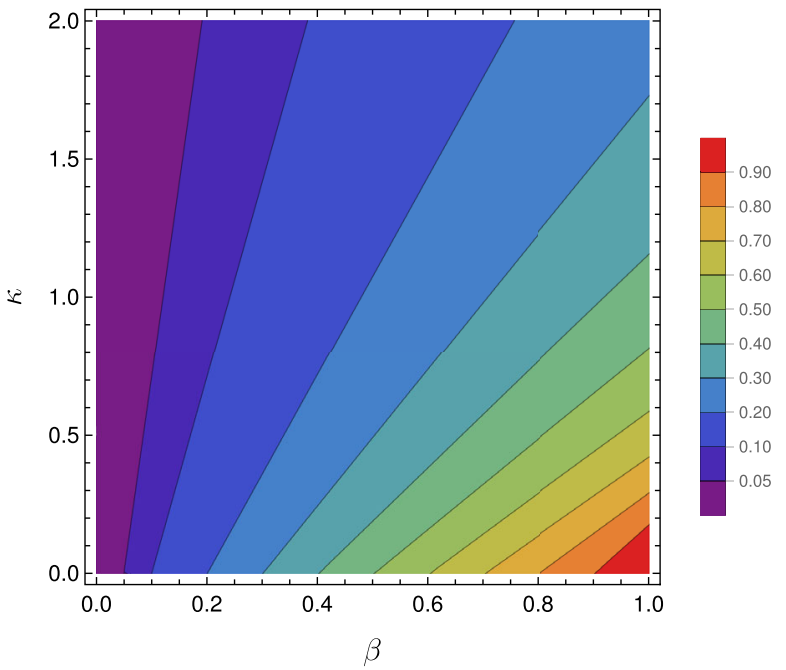

(b) Solution $y_{-}$from (36a).

FIG. 4. Exploration of the solutions $y_{ \pm}$to the end of inflation condition $\epsilon=1$. 


$$
y_{* \pm}=\operatorname{sech}\left(\operatorname{arcsech} y_{ \pm} \mp \kappa \delta_{ \pm}\right),
$$

where $\delta_{+}<\kappa^{-1} \operatorname{arcsech} y_{+}$. A choice of values $(\beta, \kappa, \delta)$ and a branch (large or small $\phi$ ) specifies the model completely. Specifically, the values for $y_{ \pm}$and $y_{* \pm}$ described above can be used to calculate the slow-roll functions at $y_{* \pm}$ and, subsequently, the values of $n_{s}$ and $r$ and the number of $e$-folds between $y_{* \pm}$ and $y_{ \pm}$. Finally, we also study the number of inflation $e$-folds produced corresponding to the different parameters, which can be obtained from Eq. (9) to be

$$
N=\frac{2 \beta\left(\frac{1}{y_{* \pm}}-\frac{1}{y_{ \pm}}\right)+(1-\beta) \ln \left(\frac{1-y_{ \pm}}{1-y_{* \pm}}\right)+(1+\beta) \ln \left(\frac{1+y_{ \pm}}{1+y_{* \pm}}\right)-2 \ln \left(\frac{y_{ \pm}}{y_{* \pm}}\right)}{4 \kappa^{2}} .
$$

Our results for the large-field solution (corresponding to $y_{-}$) with $\beta=0.5$ are encapsulated in Fig. 5. The results show a mixed degree of compatibility between the swampland conjectures and experimental data for the $S$-dual potential. The model itself can easily accommodate the experimental constraints for some region of the parameter space (namely, $\kappa \gtrsim 1$ and $\delta \lesssim 1$ ), as $n_{s}, r$, and $50 \lesssim N \lesssim 60$ are all reproducible. We can further study the compatibility between the de Sitter conjecture, the distance conjecture, and the experimental results. It is clearly visible how the bound on $\mathcal{C}$ from the de Sitter conjecture and the requirement on $\Delta \phi$ from the distance conjecture are in tension, as values of $\mathcal{C} \sim \mathcal{O}(1)$ even for the $95 \%$ C.L. lower limit on $n_{s}$ at 0.959 require $\delta \gg 1$. On the other hand, the de Sitter bound on $\mathcal{C}^{\prime}$ and the distance conjecture set bounds that get softer in the same direction of decreasing $r$. In this case, $\mathcal{C}^{\prime}$ is constrained by the data at the $95 \%$ C.L. lower limit on $n_{s}$ to $\mathcal{C}^{\prime} \gtrsim-0.02$. Despite this experimental constraint being much stronger than the $\mathcal{C} \lesssim 0.09$, there is no strong incompatibility with the distance conjecture.

As a final remark, we study the strength of Lyth's bound (11) on the current model, i.e., to which extent the model saturates such a bound. In Fig. 6, we show the value of $N C / \delta$ as obtained from Eq. (40) as a function of $\kappa$ and $\delta$, which is bound above by 1 by means of Lyth's bound. It is visible that only for small values of $\delta$ is Lyth's bound saturated.

\section{AMBIGUITY IN SLOW-ROLL PARAMETER DEFINITIONS AND IMPACT ON THE SWAMPLAND CONJECTURES}

It is common in the literature to observe two different definitions of the slow-roll parameters, one defined in terms

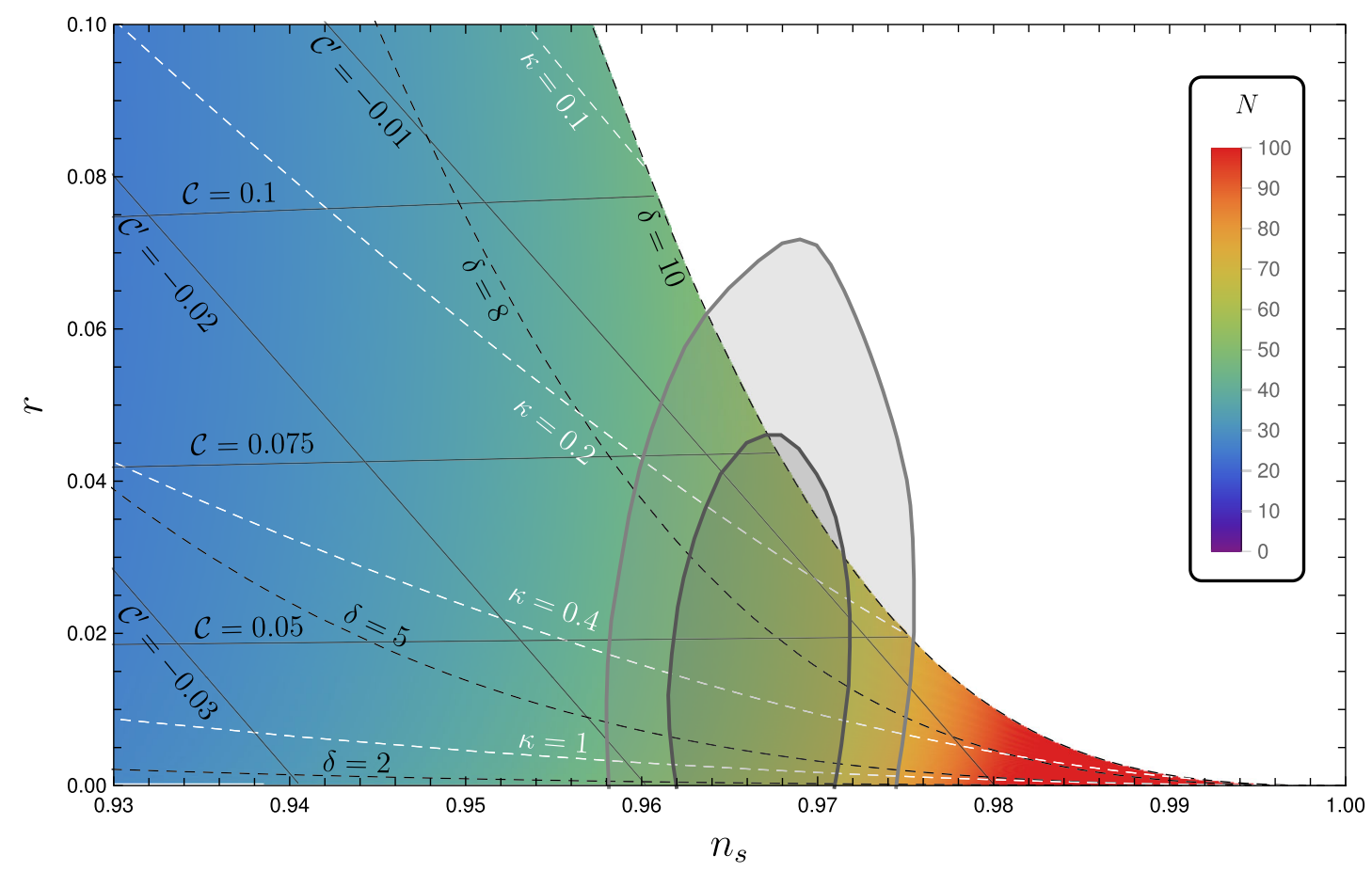

FIG. 5. Exploration of the $(\kappa, \delta)$ parameter space for a fixed $\beta=0.5$. The dashed curves in black (white) are for constant $\delta(\kappa)$. The solid lines contain the relations between $\left(\mathcal{C}, \mathcal{C}^{\prime}\right)$ and $\left(r_{s}, r\right)$ for the model [i.e., for the actual $\xi$ obtained from Eq. (31c)]. The experimental bounds are as in Fig. 1. 


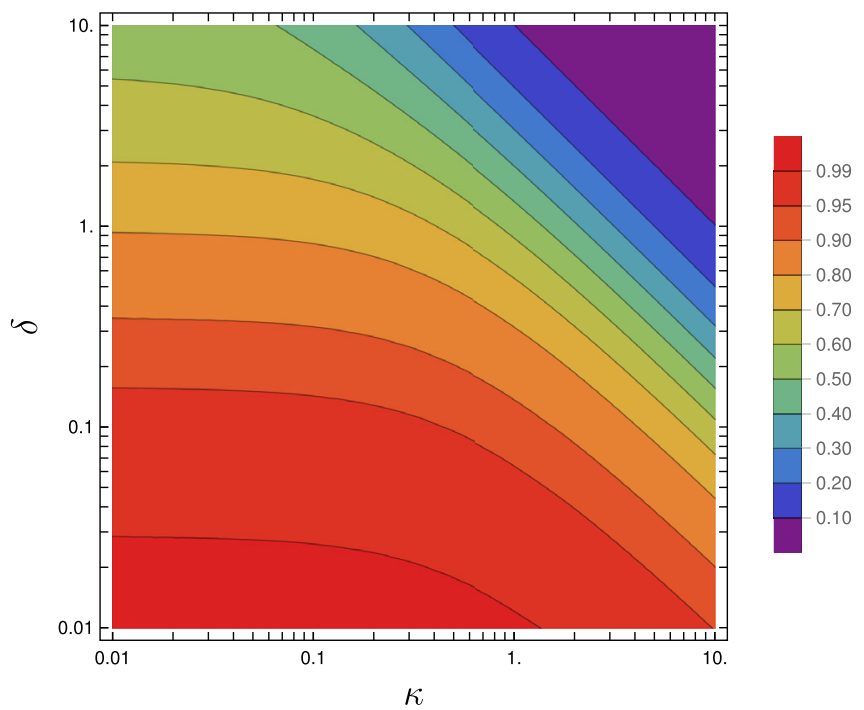

FIG. 6. Value of $N \mathcal{C} / \delta$, bound to be lower than one by Eq. (11), as a function of $(\kappa, \delta)$ for $\beta=0.5$.

of the Hubble parameter $H$ and the other in terms of the potential $V$; we have used the latter in previous sections. We have seen that the slow-roll parameters of single-field inflation defined by $V$ are in tension with the swampland conjectures. An interesting question we explore in this section is whether these two choices of parameters differ in a significant way, so that the tension with the swampland conjectures can be reduced.

Accelerated expansion occurs as long as $\ddot{a}>0$, and, since

$$
\frac{\ddot{a}}{a H^{2}}=1+\frac{\dot{H}}{H^{2}},
$$

that condition may be rewritten as $-\dot{H} / H^{2}<1$. The slowroll limit means that $H$ is constant, as this is the only way to support exponential expansion with $a=\exp (H t)$. The slow-roll regime may be considered as that in which $H$ changes slowly, which is what motivates the definition of the dimensionless slow-roll parameter as

$$
\epsilon_{H}=-\frac{\dot{H}}{H^{2}},
$$

for which $\epsilon_{H}<1$ means accelerated expansion, $\epsilon \ll 1$ means slow-roll expansion, and $\epsilon_{H}=0$ means exponential expansion. Using the Friedmann equation

$$
H^{2}=\frac{1}{3 M_{\mathrm{Pl}}^{2}}\left(V+\frac{1}{2} \dot{\phi}^{2}\right)
$$

and the equation of motion (3), this may be rewritten as

$$
\epsilon_{H}=3 \frac{\dot{\phi}^{2} / 2}{V+\dot{\phi}^{2} / 2}=2 M_{\mathrm{Pl}}^{2}\left(\frac{H_{\phi}}{H}\right)^{2}
$$

where we have made use of the relation $\dot{\phi}^{2}=-2 M_{\mathrm{Pl}}^{2} \dot{H}$. In order to connect this to the $V$ parameters, we write

$$
\frac{H_{\phi}}{H}=-\frac{3 H \dot{\phi}}{6 M_{\mathrm{Pl}}^{2} H^{2}}=\frac{V_{\phi}+\ddot{\phi}}{6 M_{\mathrm{Pl}}^{2} H^{2}}
$$

and

$$
\epsilon_{H}=\frac{M_{\mathrm{Pl}}^{2}}{2}\left(\frac{V_{\phi}+\ddot{\phi}}{V+\dot{\phi}^{2} / 2}\right)^{2}
$$

The slow-roll condition $\epsilon_{H} \ll 1$ directly implies that $V \gg \dot{\phi}^{2} / 2$, in which case

$$
\epsilon_{H} \approx \frac{M_{\mathrm{Pl}}^{2}}{2}\left(\frac{V_{\phi}+\ddot{\phi}}{V}\right)^{2} .
$$

If one further imposes the condition that $|\ddot{\phi}| \ll\left|V_{\phi}\right|$, the approximation

$$
\epsilon_{H} \approx \frac{M_{\mathrm{Pl}}^{2}}{2}\left(\frac{V_{\phi}}{V}\right)^{2}
$$

is valid. This motivates the definition of the $V$ parameter as

$$
\epsilon_{V} \equiv \frac{M_{\mathrm{Pl}}^{2}}{2}\left(\frac{V_{\phi}}{V}\right)^{2}
$$

The question of whether $\epsilon_{H}$ and $\epsilon_{V}$ may be approximately equal depends on whether the two approximations used to derive Eq. (48) are simultaneously satisfied. A glance at Eq. (44) suggests that they may not always be, as in the limit $\dot{\phi} \rightarrow 0, \epsilon_{H} \rightarrow 0$ while $\epsilon_{V}$ may take any finite value. Moreover, one can rewrite the equation of motion as

$$
\left(V_{\phi}+\ddot{\phi}\right)^{2}=\frac{3}{M_{\mathrm{Pl}}^{2}} \dot{\phi}^{2}\left(V+\dot{\phi}^{2} / 2\right)
$$

to see that a condition on the smallness of $\dot{\phi}^{2}$ does not guarantee the smallness of $\ddot{\phi}$ with respect to $V_{\phi}$ unless $V_{\phi}$ is itself small. We conclude that, in general, both conditions must be separately satisfied to guarantee the similarity between $\epsilon_{H}$ and $\epsilon_{V}$. A more comprehensive study of the differences between both parameters (as well as the secondorder ones $\eta_{H}$ and $\eta_{V}$ ) can be found in Ref. [63]. Here, we highlight only the aspects relevant for our discussion.

Given a specific potential $V(\phi)$, one can obtain a solution $\phi(t)$ to the equation of motion, subject to the initial conditions $\phi\left(t_{0}\right)=\phi_{0}$ and $\dot{\phi}\left(t_{0}\right)=\dot{\phi}_{0}$. This makes the difference between $\epsilon_{V}$ and $\epsilon_{H}$ explicit, since at $t=t_{0}$ the 


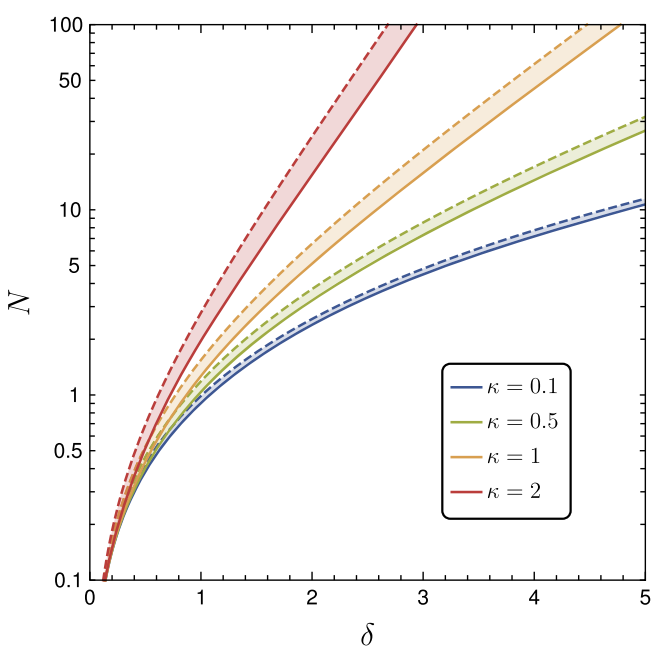

(a) $N$ as a function of $\delta$ for the $\epsilon_{H}=1$

(solid) and $\epsilon_{V}=1$ (dashed) conditions.

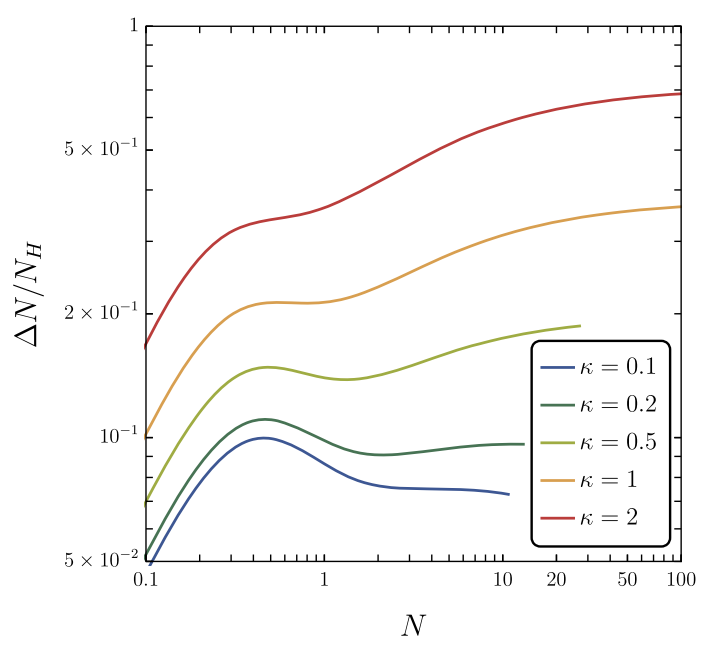

(b) Underestimation of $N$ with $\epsilon_{V}=1$

relative to $\epsilon_{H}=1$.

FIG. 7. Comparison of the two end of inflation conditions, $\epsilon_{H}=1$ and $\epsilon_{V}=1$, regarding their effect on the number of inflation $e$-folds and the parameter $\delta$.

former depends only on $\phi_{0}$ while the latter depends on both $\phi_{0}$ and $\dot{\phi}_{0}$. Therefore, the equality or similarity between $\epsilon_{V}$ and $\epsilon_{H}$ is a matter of a handpicked pair $\left(\phi_{0}, \dot{\phi}_{0}\right)$ that would guarantee both $\dot{\phi}^{2} / 2 \ll V$ and $|\ddot{\phi}| \ll V_{\phi}$.

This makes clear that the end of inflation condition $\epsilon_{H}=1$ would yield different results than $\epsilon_{V}=1$. While the latter condition is the most commonly used and is simpler to evaluate due to its sole dependence on the shape of the potential, it is the former condition that must be satisfied exactly, since it depends on the full solution of the scalar field equation of motion.

A judicious choice of initial conditions on the field and its derivative at the time at which the scale $k_{*}$ crosses the horizon should be able to accommodate multiple values of $\mathcal{C}$ or $\mathcal{C}^{\prime}$, potentially reducing the tensions with the swampland conjectures while remaining in the $H$-dictated slowroll regime. Nevertheless, keeping the de Sitter conjecture and the observed number of inflation $e$-folds under control is not guaranteed in this situation. A full study like the one presented in Sec. III, adding these initial conditions, should be considered if one aims to characterize the complete parameter space. Nevertheless, we leave that for future work, as the increase in computational complexity escapes the aim of this paper. Here, instead, we choose initial conditions that optimize the comparison between the $H$ parameters and the $V$ parameters rather than the generality of the study.

To remove part of the ambiguity caused by the freedom of choice in the initial conditions, we consider $t_{*}$ (the time at which the scale $k_{*}$ crosses the horizon) as the starting point for the solution to the equation of motion. In order to reduce the number of quantities affected by the choice of parameters, we choose to leave the observable values of $n_{s}$ and $r$ unaffected. Since these values depend on $\epsilon$ and $\eta$ at $t_{*}$, fixing the initial conditions on $\phi$ such that $\epsilon_{H}\left(t_{*}\right)=\epsilon_{V}\left(t_{*}\right)$ allows one to remove any effect of this choice on them. This is just an operational perspective that should allow us to compare the differences that $\epsilon_{H}$ and $\epsilon_{V}$ have only in regard to the other observable, $N$. This condition amounts to

$$
\dot{\phi}^{2}\left(t_{*}\right)=\frac{2 V\left(\phi_{*}\right)}{3 / \epsilon_{V}\left(\phi_{*}\right)-1},
$$

which may be rewritten in terms of $y$ as $^{3}$

$\dot{y}\left(t_{*}\right)=\frac{\sqrt{V_{0}}}{M_{\mathrm{Pl}}} \frac{2 \kappa^{2} y_{0}^{2}\left(\beta-y_{0}\right)\left(1-y_{0}^{2}\right)}{(1-\beta) \sqrt{3\left(y_{0}-\beta\right)^{2}-2 \kappa^{2} y_{0}^{2}\left(1-y_{0}^{2}\right)}}$.

In a similar manner as we proceeded before, we start by fixing the model parameters $(\beta, \kappa)$ and finding the end of inflation using the $\epsilon_{V}\left(y_{\mathrm{e}, V}\right)=1$ condition, and the value of $y_{*}$ via Eq. (39), using a given value of $\delta$, named here $\delta_{V}$. With both $y_{*}$ and $y_{\mathrm{e}, V}$, we can find the value of $N_{V}$ using Eq. (40). To quantify the difference between the choice of $\epsilon_{V}$ and of $\epsilon_{H}$, we calculate the true end of inflation through the $\epsilon_{H}\left(y_{\mathrm{e}, H}\right)=1$ condition, which provides a true value for $\delta$, as $\delta_{H}=\kappa^{-1}\left(\operatorname{arcsech} y_{*}-\operatorname{arcsech} y_{\mathrm{e}, H}\right)$, and a true number of $e$-folds $N_{H}$ as

\footnotetext{
${ }^{3}$ Only the large $\phi$ (small $y$ ) solution (the negative sign in the $y_{ \pm}$ notation) is considered here, as we deemed it to be the interesting case. Otherwise, the initial derivative should be negative.
} 
$N_{H}=-\frac{1}{M} \int_{\mathrm{Pl}} \int_{\phi_{*}}^{\phi_{e}} \frac{d \phi}{\sqrt{2 \epsilon_{H}}}=\frac{1}{\kappa} \int_{t_{*}}^{t_{\mathrm{e}, H}} \frac{\dot{y}}{y \sqrt{1-y^{2}} \sqrt{2 \epsilon_{H}}} d t$,

where $t_{\mathrm{e}, H}$ is the true time at which inflation ends.

In Fig. 7(a), we show the relation between $N_{V}$ and $\delta_{V}$, and $N_{H}$ and $\delta_{H}$. While a full analysis similar to that presented in Fig. 5 might be the only way to fully understand the relevance of the parameter set choice, here it is visible how the difference $\Delta N \equiv N_{H}-N_{V}$ grows with both $\kappa$ and $\delta$. We can see in Fig. 7(b) that for small values of $\kappa$, which are of more interest in the study of the de Sitter conjecture on $\mathcal{C}, \Delta N$ is small enough to make it irrelevant, and no significant difference would be expected in that front. Regarding $\mathcal{C}^{\prime}$, the $\mathcal{C}^{\prime} \gtrsim-0.02$ experimental bound may be accommodated a bit easier regarding the number of $e$-folds, as in that region the constraint $\epsilon_{V}=1$ is underestimating the number of $e$-folds by a few percent points of its true value.

Nevertheless, despite the minor changes introduced in relation to the swampland conjectures, Fig. 7(b) makes clear that the $\epsilon_{V}=1$ condition might end inflation too early, producing considerable underestimations of the actual number of $e$-folds.

We want to remind the reader that these results are obtained for $\beta=0.5$, as was the case with the results presented in and after Fig. 5. It must also be clarified that the information presented in Figs. 7(a) and 7(b) is not in contradiction, unlike it may seem. Figure 7(a) presents the curves $N_{H}\left(\delta_{H}\right)$ and $N_{V}\left(\delta_{V}\right)$, so the horizontal axis is not the same variable and makes it seem that, for a single $\delta$, the $V$-based condition overestimates the number of $e$-folds. Nevertheless, in Fig. 7(b), we show the curves evaluated at the same value of $\delta$. This is therefore comparing the two parameter choices for a fixed value of the field excursion $\Delta \phi$. Under this circumstance, it is clearly seen that the $V$-based conditions produce an underestimation of the number of inflation $e$-folds with respect to the $H$-based ones.

\section{CONCLUSIONS}

We have analyzed the most general form of single-field $S$-dual inflationary potentials at NLO in slow-roll parameters within the context of the swampland program and confronted model predictions with experiment. We have found that to accommodate the 95\% C.L. limit on $r<0.068$ from BICEP2/Keck Array + Planck + BAO data [11,12] we require $c \sim \mathcal{O}\left(10^{-1}\right)$. This requirement is in tension with the de Sitter conjecture. However, in the spirit of Ref. [64], we can adopt a conservative approach and regard the de Sitter conjecture as a parametric constraint where the inequality (1) holds, but the number $c$ may not be strictly $\mathcal{O}(1)$. Indeed, it is easy to establish a mass hierarchy between the lightest moduli field and inflaton to accommodate $c \sim \mathcal{O}\left(10^{-1}\right)$ [64]. From this viewpoint, constraints on inflation can then be used to constrain $c$. Still, as we have shown in Fig. 5, to accommodate $c \sim$ $\mathcal{O}\left(10^{-1}\right)$ a $\delta \sim \mathcal{O}(10)$ would be required. To be able to match such a large value of $\delta$, we must explore the subtleties of the distance conjecture, which asserts that, for any infinite field distance limit, an infinite tower of states becomes exponentially light, and, therefore, EFTs are valid only for finite scalar field variations [21-25]. This, in turn, implies a quantum gravity cutoff associated to the infinite tower of states, decreasing exponentially in terms of the proper field distance, $\Lambda_{\mathrm{QG}}=\Lambda_{\text {self }} e^{-\lambda \Delta \phi}$, where $\Lambda_{\mathrm{QG}}$ is the quantum gravity cutoff, $\Lambda_{\text {self }}$ is the cutoff of the EFT, and $\lambda$ is argued to be of the order of unity in Planck units (see, however, Refs. [38,39]). Now, since $\Lambda_{\text {self }} \leq M_{\mathrm{Pl}}$, we have $\Delta \phi \leq \lambda^{-1} \ln \left(M_{\mathrm{Pl}} / \Lambda_{\text {self }}\right)$, which indicates that the maximum field variation actually depends on the cutoff of the EFT [65]. We know that, for the EFT to describe inflation, its cutoff must be above the Hubble scale, i.e., $\Lambda_{\text {self }}>H$. If we adopt the conservative bound $\Lambda_{\text {self }} \sim H$, then $\Delta \phi \lesssim 10 M_{\mathrm{Pl}}$ [66]. Needless to say, it should be stressed that the EFT will likely break down (or at least get sensitive to the infinite tower) before the mass of the first state becomes of the order of Hubble, so the constraints might be stronger than those derived from the assumption $\Lambda_{\text {self }} \sim H$. Next-generation CMB satellites searching for primordial B modes (e.g., PIXIE [67], CORE [68], and LiteBIRD [69]) will reach a 95\% C.L. sensitivity of $r<0.002$. This will allow discrimination between smallfield $\Delta \phi<M_{\mathrm{Pl}}$ and large-field $\Delta \phi>M_{\mathrm{Pl}}$ inflationary models and will provide a final verdict for the ideas presented and discussed in this paper.

As a final remark, it would be interesting to study the full parameter space using the $H$ parameters introduced in Sec. IV rather than the $V$ parameters. As stated there, the increase in the number of free parameters would make it more feasible to reduce the tension with the swampland conjectures. An analysis like the one presented here in which the $H$ parameters are used in full is left for future work.

\section{ACKNOWLEDGMENTS}

The work of L. A. A. and J.F. S. is supported by the U.S. National Science Foundation (NSF Grant No. PHY1620661) and the National Aeronautics and Space Administration (NASA Grant No. 80NSSC18K0464). The research of I. A. was partially performed as International professor of the Francqui Foundation, Belgium. The work of D. L. is supported by the Origins Excellence Cluster. Any opinions, findings, and conclusions or recommendations expressed in this material are those of the authors and do not necessarily reflect the views of the NSF or NASA. 
[1] A. H. Guth, The inflationary universe: A possible solution to the horizon and flatness problems, Phys. Rev. D 23, 347 (1981).

[2] A. D. Linde, A new inflationary universe scenario: A possible solution of the horizon, flatness, homogeneity, isotropy and primordial monopole problems, Phys. Lett. 108B, 389 (1982).

[3] A. Albrecht and P. J. Steinhardt, Cosmology for Grand Unified Theories with Radiatively Induced Symmetry Breaking, Phys. Rev. Lett. 48, 1220 (1982).

[4] V. F. Mukhanov and G. V. Chibisov, Quantum fluctuations and a nonsingular universe, JETP Lett. 33, 532 (1981).

[5] S. W. Hawking, The development of irregularities in a single bubble inflationary universe, Phys. Lett. 115B, 295 (1982).

[6] A. H. Guth and S. Y. Pi, Fluctuations in the New Inflationary Universe, Phys. Rev. Lett. 49, 1110 (1982).

[7] A. A. Starobinsky, Dynamics of phase transition in the new inflationary universe scenario and generation of perturbations, Phys. Lett. 117B, 175 (1982).

[8] J. M. Bardeen, P. J. Steinhardt, and M. S. Turner, Spontaneous creation of almost scale-free density perturbations in an inflationary universe, Phys. Rev. D 28, 679 (1983).

[9] D. N. Spergel et al. (WMAP Collaboration), First year Wilkinson Microwave Anisotropy Probe (WMAP) observations: Determination of cosmological parameters, Astrophys. J. Suppl. Ser. 148, 175 (2003).

[10] E. Komatsu et al. (WMAP Collaboration), Seven-year Wilkinson Microwave Anisotropy Probe (WMAP) observations: Cosmological interpretation, Astrophys. J. Suppl. Ser. 192, 18 (2011).

[11] Y. Akrami et al. (Planck Collaboration), Planck 2018 results $\mathrm{X}$ : Constraints on inflation, Astron. Astrophys. 641, A10 (2020).

[12] P. A. R. Ade et al. (BICEP2 and Keck Array Collaborations), Constraints on Primordial Gravitational Waves Using Planck, WMAP, and New BICEP2/Keck Observations through the 2015 Season, Phys. Rev. Lett. 121, 221301 (2018).

[13] P. A. R. Ade et al. (Planck Collaboration), Planck 2015 results. XX. Constraints on inflation, Astron. Astrophys. 594, A20 (2016).

[14] C. Vafa, The string landscape and the swampland, arXiv: hep-th/0509212.

[15] C. Montonen and D. I. Olive, Magnetic monopoles as gauge particles?, Phys. Lett. 72B, 117 (1977).

[16] A. Font, L. E. Ibanez, D. Lüst, and F. Quevedo, Strongweak coupling duality and nonperturbative effects in string theory, Phys. Lett. B 249, 35 (1990).

[17] A. Sen, Strong - weak coupling duality in four-dimensional string theory, Int. J. Mod. Phys. A 09, 3707 (1994).

[18] T. D. Brennan, F. Carta, and C. Vafa, The string landscape, the swampland, and the missing corner, Proc. Sci., TASI2017 (2017) 015 [arXiv:1711.00864].

[19] E. Palti, The swampland: Introduction and review, Fortschr. Phys. 67, 1900037 (2019).

[20] N. Arkani-Hamed, L. Motl, A. Nicolis, and C. Vafa, The string landscape, black holes and gravity as the weakest force, J. High Energy Phys. 06 (2007) 060.

[21] H. Ooguri and C. Vafa, On the geometry of the string landscape and the swampland, Nucl. Phys. B766, 21 (2007).
[22] D. Klaewer and E. Palti, Super-Planckian spatial field variations and quantum gravity, J. High Energy Phys. 01 (2017) 088.

[23] H. Ooguri, E. Palti, G. Shiu, and C. Vafa, Distance and de Sitter conjectures on the swampland, Phys. Lett. B 788, 180 (2019).

[24] T. W. Grimm, E. Palti, and I. Valenzuela, Infinite distances in field space and massless towers of states, J. High Energy Phys. 08 (2018) 143.

[25] B. Heidenreich, M. Reece, and T. Rudelius, Emergence of Weak Coupling at Large Distance in Quantum Gravity, Phys. Rev. Lett. 121, 051601 (2018).

[26] H. Ooguri and C. Vafa, Non-supersymmetric AdS and the swampland, Adv. Theor. Math. Phys. 21, 1787 (2017).

[27] E. Palti, The weak gravity conjecture and scalar fields, J. High Energy Phys. 08 (2017) 034.

[28] G. Obied, H. Ooguri, L. Spodyneiko, and C. Vafa, de Sitter space and the swampland, arXiv:1806.08362.

[29] D. Andriot, On the de Sitter swampland criterion, Phys. Lett. B 785, 570 (2018).

[30] A. Kehagias and A. Riotto, A note on inflation and the swampland, Fortschr. Phys. 66, 1800052 (2018).

[31] S. Cecotti and C. Vafa, Theta-problem and the string swampland, arXiv:1808.03483.

[32] D. Klaewer, D. Lüst, and E. Palti, A spin-2 conjecture on the swampland, Fortschr. Phys. 67, 1800102 (2019).

[33] J. J. Heckman and C. Vafa, Fine tuning, sequestering, and the swampland, Phys. Lett. B 798, 135004 (2019).

[34] D. Lüst, E. Palti, and C. Vafa, AdS and the swampland, Phys. Lett. B 797, 134867 (2019).

[35] A. Bedroya and C. Vafa, Trans-Planckian censorship and the swampland, J. High Energy Phys. 09 (2020) 123.

[36] A. Kehagias, D. Lüst, and S. Lüst, Swampland, gradient flow and infinite distance, J. High Energy Phys. 04 (2020) 170 .

[37] R. Blumenhagen, M. Brinkmann, and A. Makridou, Quantum log-corrections to swampland conjectures, J. High Energy Phys. 02 (2020) 064.

[38] D. Andriot, N. Cribiori, and D. Erkinger, The web of swampland conjectures and the TCC bound, J. High Energy Phys. 07 (2020) 162.

[39] N. Gendler and I. Valenzuela, Merging the weak gravity and distance conjectures using BPS extremal black holes, J. High Energy Phys. 01 (2021) 176.

[40] Q. Bonnefoy, L. Ciambelli, D. Lüst, and S. Lüst, The swampland at large number of space-time dimensions, arXiv:2011.06610.

[41] E. Perlmutter, L. Rastelli, C. Vafa, and I. Valenzuela, A CFT distance conjecture, arXiv:2011.10040.

[42] M. Lüben, D. Lüst, and A. R. Metidieri, The black hole entropy distance conjecture and black hole evaporation, Fortschr. Phys. 69, 2000130 (2021).

[43] J. Calderón-Infante, A. M. Uranga, and I. Valenzuela, The convex hull swampland distance conjecture and bounds on non-geodesics, J. High Energy Phys. 03 (2021) 299.

[44] E. W. Kolb, A. J. Long, and E. McDonough, Catastrophic production of slow gravitinos, arXiv:2102.10113.

[45] D. H. Lyth, What Would We Learn by Detecting a Gravitational Wave Signal in the Cosmic Microwave Background Anisotropy?, Phys. Rev. Lett. 78, 1861 (1997). 
[46] P. Agrawal, G. Obied, P. J. Steinhardt, and C. Vafa, On the cosmological implications of the string swampland, Phys. Lett. B 784, 271 (2018).

[47] A. Achúcarro and G. A. Palma, The string swampland constraints require multi-field inflation, J. Cosmol. Astropart. Phys. 02 (2019) 041.

[48] S. K. Garg and C. Krishnan, Bounds on slow roll and the de Sitter swampland, J. High Energy Phys. 11 (2019) 075 .

[49] I. Ben-Dayan, Draining the swampland, Phys. Rev. D 99, 101301 (2019).

[50] W. H. Kinney, S. Vagnozzi, and L. Visinelli, The zoo plot meets the swampland: Mutual (in)consistency of singlefield inflation, string conjectures, and cosmological data, Classical Quantum Gravity 36, 117001 (2019).

[51] H. Fukuda, R. Saito, S. Shirai, and M. Yamazaki, Phenomenological consequences of the refined swampland conjecture, Phys. Rev. D 99, 083520 (2019).

[52] S. K. Garg, C. Krishnan, and M. Zaid Zaz, Bounds on slow roll at the boundary of the landscape, J. High Energy Phys. 03 (2019) 029.

[53] P. Agrawal and G. Obied, Dark energy and the refined de Sitter conjecture, J. High Energy Phys. 06 (2019) 103.

[54] C. I. Chiang, J. M. Leedom, and H. Murayama, What does inflation say about dark energy given the swampland conjectures?, Phys. Rev. D 100, 043505 (2019).

[55] E. W. Kolb and S. L. Vadas, Relating spectral indices to tensor and scalar amplitudes in inflation, Phys. Rev. D 50, 2479 (1994).

[56] S. Dodelson, W. H. Kinney, and E. W. Kolb, Cosmic microwave background measurements can discriminate among inflation models, Phys. Rev. D 56, 3207 (1997).

[57] J. E. Lidsey, A. R. Liddle, E. W. Kolb, E. J. Copeland, T. Barreiro, and M. Abney, Reconstructing the inflation potential: An overview, Rev. Mod. Phys. 69, 373 (1997).
[58] G. N. Remmen and S. M. Carroll, How many $e$-folds should we expect from high-scale inflation?, Phys. Rev. D 90, 063517 (2014).

[59] S. M. Leach, A. R. Liddle, J. Martin, and D. J. Schwarz, Cosmological parameter estimation and the inflationary cosmology, Phys. Rev. D 66, 023515 (2002).

[60] L. A. Anchordoqui, V. Barger, H. Goldberg, X. Huang, and D. Marfatia, S-dual inflation: BICEP2 data without unlikeliness, Phys. Lett. B 734, 134 (2014).

[61] A. Ijjas, P. J. Steinhardt, and A. Loeb, Inflationary paradigm in trouble after Planck2013, Phys. Lett. B 723, 261 (2013).

[62] J.-P. Tignol, Galois' Theory of Algebraic Equations (World Scientific, Singapore, 2001).

[63] A. R. Liddle, P. Parsons, and J. D. Barrow, Formalizing the slow-roll approximation in inflation, Phys. Rev. D 50, 7222 (1994).

[64] M. Dias, J. Frazer, A. Retolaza, and A. Westphal, Primordial gravitational waves and the swampland, Fortschr. Phys. 67, 1800063 (2019).

[65] M. van Beest, J. Calderón-Infante, D. Mirfendereski, and I. Valenzuela, Lectures on the swampland program in string compactifications, arXiv:2102.01111.

[66] M. Scalisi and I. Valenzuela, Swampland distance conjecture, inflation and $\alpha$-attractors, J. High Energy Phys. 08 (2019) 160.

[67] R. Khatri and R. A. Sunyaev, Forecasts for CMB $\mu$ and $i$-type spectral distortion constraints on the primordial power spectrum on scales $8 \lesssim k \lesssim 10^{4} \mathrm{Mpc}^{-1}$ with the future Pixie-like experiments, J. Cosmol. Astropart. Phys. 06 (2013) 026.

[68] F. Finelli et al. (CORE Collaboration), Exploring cosmic origins with CORE: Inflation, J. Cosmol. Astropart. Phys. 04 (2018) 016.

[69] M. Hazumi et al., LiteBIRD: A satellite for the studies of B-mode polarization and inflation from cosmic background radiation detection, J. Low Temp. Phys. 194, 443 (2019). 